\title{
W. Kirch
}

\section{Journal of Public Health/Zeitschrift für Gesundheitswissenschaften jetzt im Springer-Verlag Heidelberg}

Ab Beginn des Jahres 2004 erscheint die bislang vom Juventa-Verlag Weinheim herausgegebene Zeitschrift für Gesundheitswissenschaften (Journal of Public Health) im 12. Jahrgang beim Springer-Verlag. Die Zeitschrift wird Journal of Public Health heißen und den Untertitel Zeitschrift für Gesundheitswissenschaften tragen. Dies weist darauf hin, dass das Journal im Hinblick auf die Akzeptanz in der Scientific Community bzw. im Hinblick auf die Öffentlichkeitswirksamkeit auf eine breitere Basis gestellt werden soll. Die Zeitschrift wird weiter bilingual in Englisch und Deutsch, aber mit 6 Heften pro Jahr erscheinen (bisher 4 Ausgaben jährlich). Unser Bestreben ist es, in den nächsten Monaten bzw. Jahren zuerst zu überwiegend, letztendlich zu ausschließlich englischsprachigen Publikationen in der Zeitschrift zu kommen. Dies erscheint uns erforderlich, um eine "globale“ Wahrnehmung der Deutschen und Europäischen Public Health Forschung zu erreichen. Der Springer-Verlag stellt zu diesem Zweck einen sog. „native speaker“ als linguistischen Editor zur Verfügung. Darüber hinaus wird vom Verlag ein Internetauftritt unseres Journals mit den darin publizierten wissenschaftlichen Beiträgen gewährleistet sowie eine Listung in gängigen Literaturdatenbanken wie den Current Contents, dem Journal Citation Report oder dem Index Medicus angestrebt, was bisher nicht der Fall war. Langfristig wird natürlich die wissenschaftliche Reputation des Journal of Public Health von der Vergabe eines Impact-Faktors abhängen, für dessen Erlangung die Voraussetzungen beim Springer-Verlag günstig sind. Die damit verbundene Aufwertung ist für Leser, Autoren, Herausgeber und den Verlag von erheblicher Bedeutung - wird doch dadurch die Qualität dessen, was im Journal publiziert wird, gewürdigt. Die Herausgeber haben aus den oben genannten Gründen den Wechsel vom Juventa- zum Springer-Verlag einstimmig befürwortet. Dieser erfolgte in einem relativ knappen zeitlichen Rahmen, was erklärt, warum im Jahr 2004 die ersten Hefte - wie auch das vorliegende zur "Gesundheitsökonomie“ — fast ausschließlich deutschsprachige Artikel enthalten.

Ich möchte Herrn und Frau Schweim vom JuventaVerlag, im Namen der Herausgeber Prof. Badura (Biele-

W. Kirch ( $)$

Managing Editor,

E-Mail: Public.Health@mailbox.tu-dresden.de feld), Prof. Henke (Berlin), Prof. Laaser (Bielefeld), Prof. Schwartz (Hannover) und Prof. Überla (München) sehr herzlich für die Arbeit danken, die sie der Zeitschrift für Gesundheitswissenschaften gewidmet haben. In den vergangenen 3 Jahren hatte ich als geschäftsführender Herausgeber die Gelegenheit, mit den beiden genannten Verlegern des Juventa-Verlags zusammenzuarbeiten und ich kann sagen, dass beide großes persönliches Interesse, Engagement und „Herzblut" in das Erscheinen unseres Journals investiert haben. Dies und das Verständnis für die Motive der Herausgeber hat letztlich dazu geführt, dass Herr Schweim vom Juventa-Verlag, Dr. Mager und Dr. Lange vom Springer-Verlag sowie das Herausgebergremium in relativ kurzer Zeit zur gütlichen Einigung im Hinblick auf den Verlagswechsel gekommen sind.

Von Seiten des geschäftsführenden Herausgebers, zukünftig als Managing Editor bezeichnet, bleibt anzumerken, dass das Manuskriptaufkommen der Zeitschrift für Gesundheitswissenschaften in den vergangenen Jahren deutlich angestiegen ist, so dass in 2002 und 2003 sowohl Beihefte als auch Supplemente des Journals herausgegeben wurden. Dies ist eine gute Basis für das Erscheinen von mindestens 6 Ausgaben der Zeitschrift pro Jahr, was-wie gesagt-für Listung und Impact-Faktorvergabe eine der essentiellen Voraussetzungen ist. Wir werden von Zeit zu Zeit weiter Hefte mit Schwerpunktthemen herausgeben, z. B. zum Public Health-/Versorgungsforschungskongress im Juni 2004 in Bielefeld mit dem Thema „Versorgungsforschung“. Die Rejektionsrate für die in der Zeitschrift eingereichten wissenschaftlichen Manuskripte beträgt derzeit etwa $30 \%$. Schließlich hoffe ich, dass meine Mitarbeiter, derzeit federführend Frau Päd. M.A. Nicole Wagner, weiter engagiert und kompetent bei der Vorbereitung der einzelnen Journalausgaben behilflich sind, wie dies auch in den vergangenen Jahren der Fall war. Letztlich erwarte ich, dass die Neuausrichtung des Journal of Public Health einen Beitrag zur Entwicklung von Public Health in Forschung und Praxis in Deutschland bzw. im europäischen Raum leistet. Dies war das Ziel der Aktivitäten im Hinblick auf den Verlagswechsel unserer Zeitschrift.

Im Januar 2004

Prof. Dr. med. Dr. med. dent. W. Kirch 